\title{
Peer-teaching in the Secondary Music Ensemble
}

\author{
Erik Johnson \\ Correspondence: Erik Johnson, Music Education, Colorado State University, Fort Collins, Colorado, USA
}

Received: June 1, 2015 Accepted: June 16, 2015 Online Published: June 25, 2015

doi:10.11114/jets.v3i5.906

URL: http://dx.doi.org/10.11114/jets.v3i5.906

\begin{abstract}
Peer-teaching is an instructional technique that has been used by teachers world-wide to successfully engage, exercise and deepen student learning. Yet, in some instances, teachers find the application of peer-teaching in large music ensembles at the secondary level to be daunting. This article is meant to be a practical resource for secondary school music teachers to incorporate peer-teaching into their classroom. Additionally, several philosophical angles associated with peer-teaching are presented for teachers to consider including the theoretical underpinnings of collaborative learning, the cultural conceptualization of "struggle to learn," and the suggested degree structure in peer-assisted learning exercises.
\end{abstract}

Keywords: peer-teaching, peer-assisted learning, secondary music, large ensembles

\section{Introduction}

One of the highest levels of learning is teaching. This sentiment - something that many of our culture's best educators know through experience - is not an entirely new idea. John Dewey claimed that for students to learn, "the educational environment must enable individuals to liberate and organize their capacities [emphasis original]" (Dewey, 1916: $\mathrm{p}$. 127). Moreover, educational thinkers including Jean Piaget, Lev Vygotsky, Johann Pestalozzi, and even Aristotle expressed the belief that when an individual wrestles with the process of organizing what they know and teach it to another, their own depth and complexity of understanding is greatly expanded.

Individual knowledge that develops when students teach what they know to a peer can be beneficial to both the person who receives and for the one who shares. The following quote by educational scholar Audrey Gartner captures the sentiment of teaching being a powerful form of learning (Gartner, 1998: p. 281):

"We learn:

$20 \%$ of what we READ

$30 \%$ of what we HEAR

$40 \%$ of what we SEE

$50 \%$ of what we both SEE and HEAR

$70 \%$ of what we DISCUSS with others

$80 \%$ of what we EXPERIENCE personally

$90 \%$ of what we TEACH others."

According to collaborative learning experts Keith Topping and Stewart Ehly, peer-assisted learning - an instructional arrangement where peers help each other to gain knowledge and skill through active help and support - entails individuals sharing knowledge until it becomes a common possession (Topping \& Ehly, 2001). Many researchers in music education have presented evidence that peer-assisted learning (PAL) often leads to meaningful gains in music achievement, student engagement, self-concept, and motivation to learn (Alexander \& Dorrow, 1983; Darrow, Gibbs, Wedel, 2005; Goodrich, 2007; Johnson, 2011; Johnson, 2013). Additionally, PAL is a way for teachers to create a community of learners where the "open flow of ideas," the "faith in the individual and the collective," and the use of "critical reflection to evaluate problems," are all cultivated aspects of student experience. Moreover, educational scholars have noted that these aspects of PAL also help to promote the ideals of democracy in education (Apple \& Beane, 1995).

Fostering the values of democracy in education through PAL can help students to become the architects of their own 
education. In conjunction with teacher-led education, PAL opens a structured space for students to assume a higher degree of responsibility for their own learning. Through working in collaborative pairs or small groups, students can practice and hone face-to-face interaction, positive interdependence, knowledge co-construction, interpersonal skills, and assessment of group and individual learning effectiveness. PAL also provides a path for students to practice the social values of a democratic society by providing a structured, collaborative vehicle to enact shared goals, individual choice, reflective thought, and the development of awareness for individual responsibilities that can contribute to the public good. The power of PAL, however, becomes most apparent when students struggle through road blocks in their own understanding, even if that means that they do not always arrive at a 100\% correct solution or answer. Beyond simply making gains in achievement, PAL cultivates an awareness and empathy for learning because of the intellectual struggle that is encountered.

Given that there are those who criticize PAL as a pooling of ignorance, a good and not entirely unreasonable question is how are students supposed to teach each other when their knowledge is incomplete? Guided by the assumption that gaining knowledge is a process of unpacking and rebuilding what is known through individual and collective reasoning, PAL offers the opportunity for students to refine their knowledge by encountering what they do not know. Refinement becomes possible as individuals encounter potential misconceptions or lack of knowledge in one of three ways: 1) through discussion and reasoning with a peer whereby knowledge and effort is pooled, 2) reasoning, reconstructing, deconstructing, and reconciling knowledge when attempting to complete a shared task, or 3) through intermittent, student-initiated efforts to seek outside resources to resolve misconceptions. However, for PAL to be successful, teachers must consider important differences between students who work together, specifically differences in cognitive ability and social status that have been found to influence the process of how individuals learn from each other.

\section{The Process of Students Learning Together}

Up until the early 1980's, PAL was primarily utilized as a way for more experienced and able students to increase the performance of less able students. In music classrooms, a clear example of this has been the utilization of student section leaders who in many instances lead sectionals and establish section norms. In this arrangement, learning can be conceptualized as what Lev Vygotsky labeled the zone of proximal development - the distance between what an individual can achieve through independent problem solving versus what is possible when engaging in mutual goal-directed activity with a more advanced individual (Vygotsky, 1978). Hence, the knowledge of the more advanced learner and the social dynamic are thought to be the catalysts for learning in this scenario.

While this arrangement has been useful for developing student leadership, of greater interest is the potential for learning that happens when students who have similar abilities teach and learn from one another. Learning in this context is more akin to what Jean Piaget described as cognitive conflict - a process where students wrestle with concepts that are confusing in an effort to ultimately achieve understanding (Piaget, 1952). According to Piaget, the result of cognitive conflict is either the assimilation of new information that reinforces previously held understandings, or the accommodation of completely new information to incite mental growth. In PAL, cognitive conflict is often observed when students of like ability experience similar conceptual confusion. However, counterproductive to resolving cognitive conflict, it is often the case that when students encounter a problem the teacher takes over and tells the student the right answer. In this scenario, students are denied a valuable learning space for collaboration to take place whereby they are given the time to explore misunderstandings and come up with their own solution.

Recently, teachers and scholars in music have been exploring the learning effectiveness of two scenarios: what happens when students of like ability and of different abilities teach each other in a reciprocal fashion (i.e., play both the part of the helper and the helped). Educational researchers have highlighted that reciprocal PAL - situations where students switch back and forth between the teacher and the student - often produces higher achievement than when student roles are fixed (i.e., only one student plays the role of the teacher) (Ginsburg-Block, Rohrbeck, \& Fantuzzo, 2006). Interestingly, when investigating reciprocal PAL in secondary music classrooms, strong achievement gains have been found for both like ability and of divergent ability pairs (Johnson, 2013). Ultimately, the fact that reciprocal PAL has been shown to be more effective than fixed-role PAL and that both similar and divergent ability pairing result in achievement gains is good news for teachers who want to choose the approach that best serves their students' needs.

\subsection{Social Considerations when Pairing Students Together}

Recent research with students in secondary large ensembles has revealed that the level of a student's individual socioeconomic status (SES) may also impact learning in PAL arrangements. While students of low SES do well in both similar and divergent ability pairings, as SES rises student achievement tends to increase only when working with a peer of like ability (Johnson, 2013). One possible explanation is that when the difference in ability between individuals is great, students may have more difficulty working well together because they don't want to expose their weaknesses. However, sociocultural educational researchers suggest that this can be especially true for students of mid to high SES 
who may be more enculturated to conceptualize intellectual struggle as a weakness (Li, 2005; Payne, 1998).

\subsection{Re-conceptualizing Struggle to Learn}

Educational scholar Jin Li claims that Western values often emphasize the virtues of mental ability and creativity. In a classroom where these virtues are highlighted, learning is often framed as a task to be tackled where struggle to grasp a concept is seen as an individual weakness. Hence, it is necessary for teachers who use PAL to acclimate their students to the idea that intellectual struggle is supposed to happen and is a necessary precursor to meaningful learning. Moreover, teachers must outline what a full unfolding of cognitive conflict looks like to their students so that accommodation and acquisition can run its full course, free of unnecessary self-doubt. When students feel comfortable saying, "I don't know" to a peer who is committed to finding a solution, individual engagement, motivation and self-concept can benefit because students have a partner to tackle the struggle with.

\subsection{Degree of Structure}

The degree to which teachers control the learning environment has also been found to impact student achievement when using PAL. Specifically, achievement gains are often greater in situations when students determine their own rules for interaction and decide how much material is to be studied in each session than when situations are more tightly controlled. However, it is important that teachers find a balance between structured guidance while still allowing the autonomy necessary for students to organize their own capacities. Students who need more structure will initially rely on teacher guidance while students who are more ready for a challenge can assume more responsibility for their own path.

It is also important for teachers to consider that learning between peers of similar status and ability may not look or feel like the learning they are used to seeing during traditional instruction. However, before sweating about the chaos that can ensue when students are left to their own devices, teachers are well served to help students ahead of time by providing a list of problem-solving options that they can choose from to help reason through situations when neither they or their partner know how to proceed. Several strategies to help students through this process are listed below in the section on student training (see Tables 2 and 3).

\section{Sample PAL Units for the Large Ensemble Classroom}

A variety of cognitive and performance-based musical outcomes have been associated with PAL in the secondary music classroom. This list includes sight-reading, composition, improvisation, listening, and an understanding of music theory. To put a system into place, however, takes a great deal of planning on the part of the teacher. Producing or obtaining instructional materials, the training of the students, and identifying benchmarks for assessment are among the details that need to be considered. To assist teachers with beginning this process, listed below in Table 1 are six different flexible musical outcomes that can be used in the secondary large ensemble classroom including suggested procedures for implementation organized by short, medium, and long-term projects. 
Table 1. Sample PAL units with large ensembles

Short, medium, and long-term projects

Short-Term Projects (2-3 weeks)

Scenario One

Objective: Students will be able to interpret (verbally and in writing) dotted and tied rhythms in common time

Scenario Two

Objective: Students will be able to draw the circle of 5 ths for major key signatures from memory

Procedures for Short-Term Projects

Step 1: Distribute, administer, and evaluate a diagnostic worksheet for desired outcome.

Step 2: Pair students of like ability together (based upon diagnostic assessment).

Step 3: In one, 15-minute training session, have students determine goals and rules for interaction.

Step 4: Students work together every other day for 15 minutes.

Step 4a: Teacher demonstrates performance of the objective for students at the end of each session.

Step 5: At the end of session 5 or 6, students complete a summative project post-test together (grade is based partially or entirely upon amount of combined improvement from diagnostic test).

\section{Medium-Term Projects (4-9 weeks)}

Scenario Three

Objective: Students will be able to utilize the elements of form, harmony (I, IV(ii), V7), melody and accompaniment, rhythm (4/4 or 6/8), dynamic contrast, and articulation within a restricted range to compose a 32-measure duet in rounded binary form (ABAA).

Scenario Four

Objective: Students will be able to transcribe a short pre-recorded audio excerpt using traditional notation, perform their transcription, and reflect in writing upon the differences in their performance and the original audio recording.

Procedures for Medium-Term Projects

Step 1: Teacher determines student pairings based upon personality, ability, and instrumentation.

Step 2: Teacher trains all students how to engage in peer-teaching by guiding two student volunteers through a shortened version of the project in three, 20-minute class sessions over the course of one week.

Step 2a: In session one, the teacher orients the students to the project and has students determine individual goals and rules for interaction for their pair.

Step $2 \mathrm{~b}$ : In session two, with the help of the entire class, the teacher guides the volunteer students to understand the scope of the project. Through the demonstration of the volunteers, the teacher has all students engage with the specific conceptual elements to be used as part of the project.

Step 2c: In session three, with the help of the entire class, the teacher helps the two volunteer students complete a mini version of the entire project (e.g., for composition, students will harmonically map the form for 32 bars by including basic harmony and voicing then compose the first 4 bars of the duet using interlocking rhythms between the two voices).

Step 3: Student pairs work together for 15-20 minutes twice a week for 3-8 weeks.

Step 3a: During the project mid-point, students share their "in-progress" work with the class while the teacher and other students provide constructive feedback.

Step 4: During the final week, students polish their projects then record and post their projects to a closed YouTube channel.

Step 5: In one, 15-minute session, student pairs meet together one last time to reflect upon goals and to complete confidential self and peer evaluation forms.

\section{Long-Term Projects (10 weeks to Full Semester)}

Scenario Five

Objective: Students will be able to successfully memorize and perform a large ensemble work alone and with others.

Scenario Six

Objective: Students will be able to successfully perform all of their major scales and arpeggios (one octave) in $8^{\text {th }}$ notes $(92$ bpm) with minimal reference to written notation (e.g., they can reference the circle of 5 ths).

Procedures for Long-Term Projects

Step 1: With teacher assistance, students choose their own partners.

Step 2: Teacher leads students through one, 30-minute training session where students work together to determine short, medium, and long-term individual and partner-specific goals in addition to rules they will follow for collaborative interaction.

Step 3: Student pairs (or small groups such as sections of the ensemble) work together for 15-minutes twice a week for two weeks.

Step 4: During week three (or after students have met together 3-4 times), the teacher leads students through one, 20-minute group discussion about the differences between cooperative and collaborative learning, individual work and positive interdependence, and has student pairs meet together for 10 minutes to refine/re-define group and individual mid to long-term goals.

Step 5: Student pairs (or small groups) work together for 15-minutes twice a week for 4 weeks. During this time, students will work on breaking down and refining specific passages/phrases, writing in counts for tricky rhythms, pinpointing problematic intonation, and focus upon articulation/diction.

Step 6: During week 6, student groups combine to form medium sized groups of 6-8 participants. Over the course of the next two sessions, students individually perform for group members and receive written formative feedback and reflect (in writing) upon the progress they have made toward achieving their goals.

Step 7: Students return to work in their original groups. Students individually complete formative performance tests for teacher and review short, medium, and long-term group goals with the teacher.

Step 8: Student pairs (or small groups) work together for 15-minutes twice a week for 4 weeks and continue to break down and refine their performance goals. Students practice and familiarize themselves with the process of electronically recording their performance.

Step 9: Students individually record their final performances and electronically submit to teacher for summative project grade (summative grade is $1 / 2$ individual score, $1 / 4$ the percentage increase from formative performance test [step 7], and 1/4 of the average of their group performance). 


\section{Student Training}

For successful reciprocal PAL to occur, it is essential for teachers to train students how to use reciprocal peer teaching techniques. Teacher-led training sessions can vary in length, however, training should emphasize both interdependence (i.e., willingness to help their partner) and intersubjectivity (i.e., the process of creating meaning together). Additionally, it is important for teachers to guide student pairs in the creation of productive rules and processes. Below in Table 2 are some suggestions to help teachers implement effective student training sessions.

Table 2. Sample PAL training strategies

Training strategies $1-4$

Training Strategy \#1 - Modeling the "Reciprocal Interaction Procedure"

To teach students how to utilize the steps listed below, choose a short goal that is connected to the project objective and relatively easy to achieve. The steps below should be available for students to see. Lastly, the teacher guides the student pair through each of the following steps.

Step One: Show and Tell (Cognitive Modeling)

One peer verbalizes while performing the task and the other observes

Example: Watch while I write in the counts and count this measure out loud (even if I don't completely understand how to do it). Before I count, I will tell you how I'm going to do it.

Step Two: Try it Together (Overt Guidance)

One peer performs with the guidance of the other peer's instruction

Example: Both students work together to write in the counts and count the rhythm out loud. Work together until you both have figured out something new. Sink or Swim together!

Step Three: Speak Aloud Alone (Overt Self-Guidance)

One peer performs while instructing him or herself aloud (opposite from step one - the peer who observed now verbalizes the task) Example: On my own, I am going to talk my way through each step while I do it. The other student watches and corrects any mistakes afterward.

Step Four: How did I do? (Direct Feedback)

One peer (observer in step 3) provides feedback to their peer based upon their performance and repeats steps above if necessary

Step Five: Ask the Teacher for Help (seeking expert knowledge/tools)

Once student pairs have gone through the first four steps at least twice, they may ask the teacher for help or seek out another resource Example: The students both feel like they are getting a lot better but still have some unanswered questions.

Training Strategy \#2 - Task Demonstration and Clear Explanation

Entire class goes through a "practice problem" associated with project objective with their assigned partner referencing the procedure listed above.

\section{Training Strategy \#3 - Task Prompting and Imitation}

Entire class is presented a problem without receiving any other information and has to reason through to an answer with their partner. At the conclusion of this task, teacher will ask students to share what techniques they used (for benefit of entire class).

Training Strategy \#4 - Identifying Patterns of Error

Each pair goes through a simple exercise (e.g., identifying flats/sharps on the staff) where one partner makes a mistake and the other partner needs to identify the mistake. At the conclusion of the task, the teacher will provide the correct answer on the board while students reflect upon how they knew there was an error.

Training Strategy \#5 - Positive Cycles of Instruction

The teacher models being one of the students in the reciprocal pair while using a Praise-Suggestion-Praise or Pause-Prompt-Praise feedback cycle.

4.1 Practicing Interactions

After students have been paired together, teachers can provide student training in 1-3 short 20-30 minute full-group sessions. Below are five different strategies that can be used to effectively train large groups of students. For best results, teachers need to select and guide two student volunteers through each of these processes in front of the class. Additionally, it is necessary for all student pairs to sit together during training sessions.

\subsection{Guiding Students to Create Rules}

Researchers have found evidence that when students have more autonomy in PAL experiences they are almost twice as successful when compared with environments that are more tightly controlled by the teacher (Rohrbeck, Ginsburg-Block, Fantuzzo, and Miller, 2003). However, the teacher does play an important role in guiding students to form rules with their partner regarding PAL interaction. The following list in Table 3 is a set of questions that teachers might ask students to answer with their partner as they seek to determine how they will interact throughout the PAL process. Also included are hypothetical answers students might come up with. 
Table 3. Training prompts for creating rules

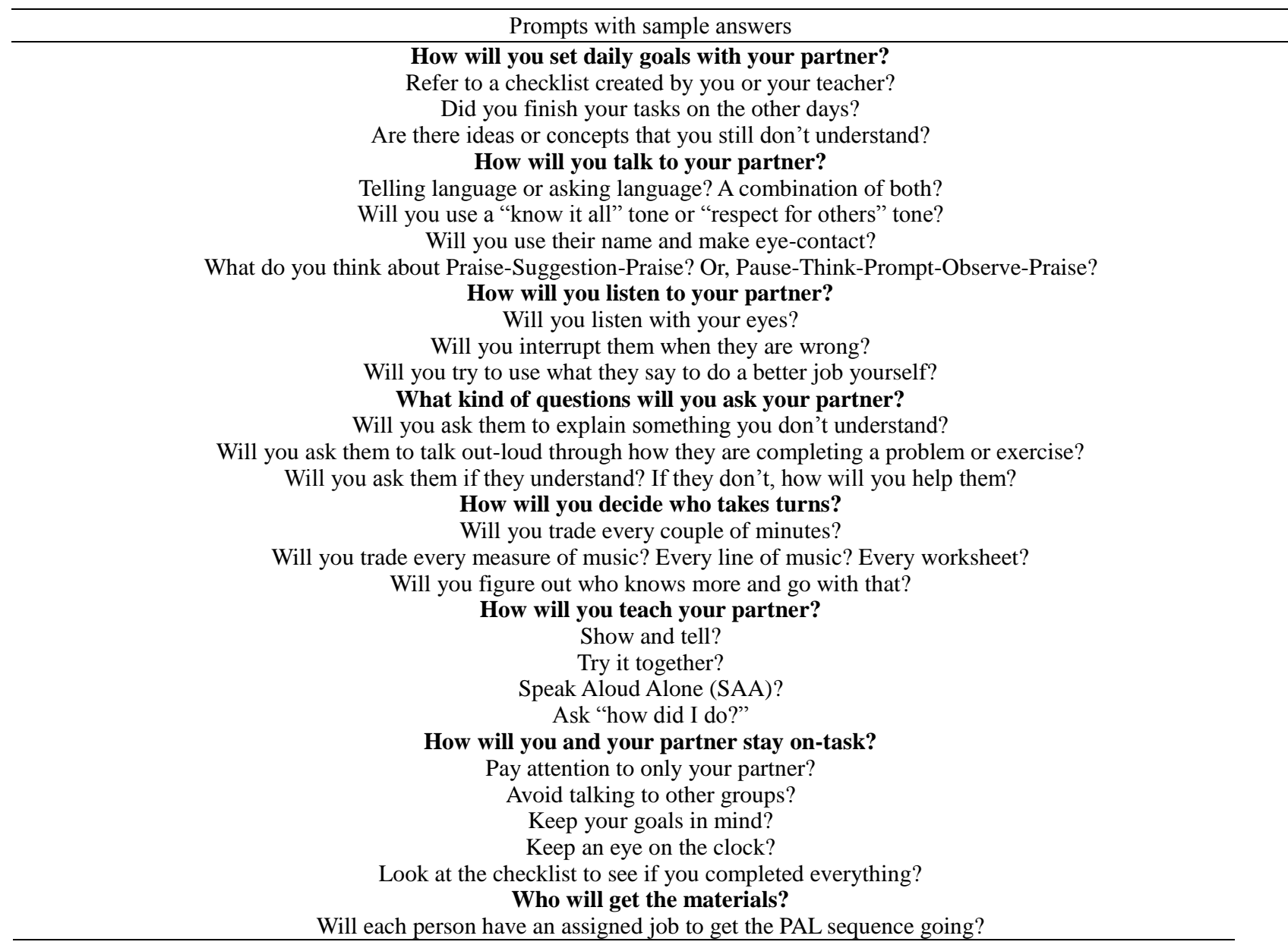

\subsection{Assessment of Student Learning}

Assessment is a necessary and valuable component of PAL for both students and teachers. For best results, teachers should utilize multiple forms of assessment during the duration of the project including diagnostic, formative, and summative means of gathering information regarding student learning.

\subsubsection{Diagnostic Assessment}

Diagnostic assessment in PAL should be low-stakes and take the form of written worksheets or recordings of students performing short and simple excerpts related to the project objective if the desired outcome is a musical performance task. The use of diagnostic assessment can be used to place students in like or divergent ability pairs or simply help orient the students to a new set of competencies.

\subsubsection{Formative Assessment}

Formative assessment or "in-progress" assessment in PAL should be "low-stakes" and originate primarily from students giving verbal and written feedback during the PAL process. Teachers can also use formative assessment during longer PAL projects (e.g., scenarios 5 and 6 above) to provide guidance relevant to student's individual and group progress toward achieving short and mid-range project goals.

\subsubsection{Summative Assessment}

Summative assessment or "high-stakes" assessment should be used to help students evaluate the final achievement of their individual and group goals. In PAL, summative assessment can include a combination of individual performance, the average of group performance, or the amount of individual or group progress made from initial diagnostic assessment. It is recommended that individual performance account for at least half of the summative assessment grade. However, to promote interdependence it is also necessary for a combined average of group performance to also be included (e.g., $1 / 4$ to $1 / 2$ of the total grade). While students are often individually motivated by summative assessment, when using PAL it is important to remind students that the purpose of summative assessment is to evaluate individual 
and group progress toward achieving predetermined teacher-created learning goals.

\subsection{Creating a Community Learners}

According to music education philosopher Bennett Reimer, if the ultimate goal of music education is to foster an artistic disposition, or at the very least aficionados who attend to music with mental, emotional, and physical energy, then music educators must expand the roles that students play in music classrooms (Reimer, 2003). PAL can help students experience a variety of musical roles that foster the active exploration of various musical concepts. In order to foster democratic principles in the music classroom and hold true to John Dewey's call for education to "enable individuals to liberate and organize their capacities", it is necessary for large ensemble directors to give students the opportunity to create, elucidate, articulate, and validate their own opinions and musical understandings with others. However, the teaching and learning that happens in the large ensemble context has often been historically centered on teacher-determined performance goals rather than upon a flexible learning model like PAL that allows students to meet their individual needs as independent musicians (Shively, 2004).

While the goal of bringing PAL into the large ensemble is not to diminish the rich tradition of ensemble performance in the American public schools, the learning potential of those involved can be inhibited when an ensemble leader assumes primarily an autocratic role. The danger of the teacher-conductor possessing the majority of control in the classroom is that "students are not being given the opportunity to develop the individuality and responsibility required to be an artist... while producing results quickly, this [didactic instruction] leaves students dependent on the teacher, when what is desired is the development of musical independence" (Reimer, 2000). Indeed, it can be difficult or unattractive for teachers to embrace PAL and other collaborative learning practices because of the louder and more raucous nature of student interaction as well as the 'bumpy,' or messy interactions between students when governing their own processes. Therefore, successful PAL requires that the teacher embrace a role that is more 'guide-on-the-side' and less 'sage-on-the-stage,' leaving room for students to make autonomous decisions. Moreover, teachers must tolerate environments different than those where students dutifully sit silently, awaiting the next set of instructions.

The focus of PAL is to allow students to organize their own learning experience, and to a degree, learn about or explore unknown or less developed concepts with a peer. However, with more autonomy to organize their learning environment, a higher degree of responsibility for productive learning is placed upon the individual student. While PAL looks and feels different from traditional teacher-led instruction, if students are to develop mental, emotional, and physical understandings of music, then the responsibility for developing these traits must be shared by both student and teacher. Ultimately, PAL is a way for teachers to share the responsibility for learning with the student and to foster student autonomy and agency, which over time, can lead to musical independence.

\section{References}

Alexander, L., \& Dorow, L. G. (1983). Peer tutoring effects on the music performance of tutors and tutees in beginning band classes. Journal of Research in Music Education, 31(1), 33-47. http://dx.doi.org/10.2307/3345108

Apple, M. W., \& Beane, J. A. (1995). Democratic schools. Alexandria, VA: Association for Supervision and Curriculum Development.

Darrow, A. A., Gibbs, P., \& Wedel, S. (2005). Use of classwide peer tutoring in the general music classroom. Update: Applications of Research in Music Education, 24(1), 15-26. http://dx.doi.org/10.1177/87551233050240010103

Dewey, J. (1916). Democracy and education: An Introduction to the philosophy of education. Chicago: Macmillan.

Gartner, A. (1998). Mutual tutoring: Embedding and extending peer-assisted learning. K. Topping \& S. Ehly (Eds.), Peer-Assisted Learning, 281-289. Hillsdale, NJ: Erlbaum.

Ginsburg-Block, M. D., Rohrbeck, C. A., \& Fantuzzo, J. W. (2006). A meta-analytic review of social, self- concept, and behavioral outcomes of peer-assisted learning. Journal of Educational Psychology, 98(4), 732-773. http://dx.doi.org/10.1037/0022-0663.98.4.732

Goodrich, A. (2007). Peer mentoring in a high school jazz ensemble. Journal of Research in Music Education, 55(2), 94-114. http://dx.doi.org/10.1177/002242940705500202

Johnson, E. (2011). The effect of peer-based instruction on rhythm reading achievement. Contributions to Music Education, 38(2), 25-42.

Johnson, E. (2013). The effect of symmetrical and asymmetrical peer-assisted learning structures on music achievement and learner engagement in the secondary large ensemble. Unpublished Doctoral Dissertation.University of Colorado, Boulder. 
Li, J. (2005). Mind or virtue: Western and Chinese beliefs about learning. Current Directions in Psychological Science, 14(1), 190-194. http://dx.doi.org/10.1111/j.0963-7214.2005.00362.x

Payne, R. K. (1998). A framework for understanding poverty. New York: RFT Publishers.

Piaget, J. (1952). The origins of intelligence in children. (M. Cook, trans.). New York: International Universities Press. (Originally published in 1936). http://dx.doi.org/10.1037/11494-000

Reimer, B. (2000). Performing with Understanding: The Challenge of the National Standards for Music Education. Reston, VA: Music Educators National Conference.

Reimer, B. (2003). A philosophy of music education: Advancing the vision (3rd ed.). Upper Saddle River, NJ: Prentice Hall.

Rohrbeck, C. A., Ginsburg-Block, M. D., Fantuzzo, J. W., \& Miller, T. R. (2003). Peer-assisted learning interventions with elementary school students: A meta-analytic review. Journal of Educational Psychology, 95(2), 240-267. http://dx.doi.org/10.1037/0022-0663.95.2.240

Shively, J. (2004). In the face of tradition: Questioning the roles of conductors and ensemble members in school bands, choirs, and orchestras. Questioning the music education paradigm, 11(2), 179-190.

Topping, K. J., \& Ehly, S. W. (2001). Peer assisted learning: A framework for consultation. Journal of Educational and Psychological Consultation, 12(2), 113-132. http://dx.doi.org/10.1207/S1532768XJEPC1202_03

Vygotsky, L. S. (1978). Mind in society: The development of higher psychological processes. Cambridge, MA: Harvard University Press. 